\title{
Robust Stability Criteria for Uncertain Neutral Systems with Interval Nondifferentiable Time-Varying Delay and Nonlinear Perturbations
}

\author{
W. Weera ${ }^{1,2}$ and P. Niamsup ${ }^{1,2}$ \\ ${ }^{1}$ Department of Mathematics, Chiang Mai University, Chiang Mai 50200, Thailand \\ ${ }^{2}$ Center of Excellence in Mathematics, CHE, Si Ayutthaya Road, Bangkok 10400, Thailand \\ Correspondence should be addressed to P. Niamsup, scipnmsp@chiangmai.ac.th
}

Received 7 July 2011; Accepted 13 September 2011

Academic Editor: James Buchanan

Copyright (C) 2011 W. Weera and P. Niamsup. This is an open access article distributed under the Creative Commons Attribution License, which permits unrestricted use, distribution, and reproduction in any medium, provided the original work is properly cited.

\begin{abstract}
We study the robust stability criteria for uncertain neutral systems with interval time-varying delays and time-varying nonlinear perturbations simultaneously. The constraint on the derivative of the time-varying delay is not required, which allows the time-delay to be a fast time-varying function. Based on the Lyapunov-Krasovskii theory, we derive new delay-dependent stability conditions in terms of linear matrix inequalities (LMIs) which can be solved by various available algorithms. Numerical examples are given to demonstrate that the derived conditions are much less conservative than those given in the literature.
\end{abstract}

\section{Introduction}

It is well known that the existence of time delay in a system may cause instability and oscillations. Example, of time-delay systems are chemical engineering systems, biological modeling, electrical networks, physical networks, and many others, [7-16]. The stability criteria for system with time delays can be classified into two categories: delay-independent and delay-dependent. Delay-independent criteria do not employ any information on the size of the delay; while delay-dependent criteria make use of such information at different levels. Delay-dependent stability conditions are generally less conservative than delay-independent ones especially when the delay is small.

In many practical systems, models of system are described by neutral differential equations, in which the models depend on the delays of state and state derivatives. Heat exchanges, distributed networks containing lossless transmission lines and population ecology are examples of neutral systems because of its wider application. Therefore, several researchers have studied neutral systems and provided sufficient conditions to guarantee the 
asymptotic stability of neutral time delay systems, see $[5,9,11-14,16,17]$ and references cited therein.

Well-known nonlinearities, as the delays, may cause instability and poor performance of practical systems, which have driven many researchers to study the problem of nonlinear perturbed systems with state delays during the recent years $[5,7,9,18]$. In [18], the delaydependent robust stability for linear time-varying systems with nonlinear perturbations is given, by using the Newton-Leibniz formula which has been taken into account instead of applying an integral inequality. In [7], a model transformation technique is used to deal with the stability of system with time varying for delays and nonlinear perturbations. In [9], based on a descriptor model transformation combined with a matrix decomposition approach, the robust stability of uncertain systems with time varying discrete delay is studied by applying an integral inequality. However, these model transformations often introduce additional dynamics which leads to relatively conservative results. In [5], the neutral delay and the discrete delay are all time-varying, while the derivative of discrete delay is less than 1 which limits its bigger application. In most studies the time-varying delays are required to be differentiable $[1-5,7,9,11-14,16,18]$. Therefore their methods have a conservatism which can be improved upon. However, in most cases, these conditions are difficult to satisfy. From these reasons, the conditions are interesting to study, but there are fewer results for removing restriction to the derivative of interval time-varying delays. Therefore, in this paper we will employ some new techniques so that the above conditions can be removed.

In this paper, the problem of delay-dependent criterion for asymptotic stability for uncertain neutral system is studied with interval time-varying delay and time-varying nonlinear perturbations simultaneously. The restriction to the derivative of the interval time-varying delays is removed, which means that a fast interval time-varying delay is allowed. Based on the Lyapunov-Krasovskii theory, we derive new delay-dependent stability conditions in terms of linear matrix inequalities (LMIs) which can be solved by various available algorithms. The new stability condition is much less conservative and is more general than some existing results. Numerical examples are given to illustrate the effectiveness of our theoretical results.

\section{Problem Formulation and Preliminaries}

The following notations will be used in this paper: $\mathbb{R}^{+}$denotes the set of all real nonnegative numbers; $\mathbb{R}^{n}$ denotes the $n$-dimensional space and the vector norm $\|\cdot\| ; M^{n \times r}$ denotes the space of all matrices of $(n \times r)$-dimensions. $A^{T}$ denotes the transpose of matrix $A ; A$ is symmetric if $A=A^{T}$; $I$ denotes the identity matrix; $\lambda(A)$ denotes the set of all eigenvalues of $A ; \lambda_{\max }(A)=\max \{\operatorname{Re} \lambda ; \lambda \in \lambda(A)\} . x_{t}:=\{x(t+s): s \in[-h, 0]\},\left\|x_{t}\right\|=\sup _{s \in[-h, 0]}\|x(t+s)\| ;$ $C\left([0, t], \mathbb{R}^{n}\right)$ denotes the set of all $\mathbb{R}^{n}$-valued continuous functions on $[0, t]$; Matrix $A$ is called semipositive definite $(A \geq 0)$ if $x^{T} A x \geq 0$, for all $x \in \mathbb{R}^{n} ; A$ is positive definite $(A>0)$ if $x^{T} A x>0$, for all $x \neq 0 ; A>B$ means $A-B>0$. The symmetric term in a matrix is denoted by $*$.

Consider the following neutral system with time-varying delay:

$$
\begin{gathered}
\dot{x}(t)-C \dot{x}(t-d(t))=A(t) x(t)+B(t) x(t-\tau(t))+D_{1}(t) f_{1}(t, x(t))+D_{2}(t) f_{2}(t, x(t-\tau(t))), \\
x\left(t_{0}+\theta\right)=\phi(\theta), \quad \theta \in[-h, 0]
\end{gathered}
$$


where $x(t) \in R^{n}$ is the state vector, $d(t)$ is a neutral delay, $\tau(t)$ is a time-varying continuous function which satisfies

$$
0 \leq \tau_{m} \leq \tau(t) \leq \tau_{M}, \quad 0 \leq d(t) \leq d, \quad \dot{d}(t) \leq \delta,
$$

where $\tau_{m}, \tau_{M}, d, \delta$ are constants and $h=\max \left\{d, \tau_{M}\right\}$; the initial condition function $\phi(t)$ denotes a continuous vector-valued initial function of $t \in[-h, 0], f_{1}(t, x(t))$ and $f_{2}(t, x(t-$ $\tau(t)))$ are unknown nonlinear perturbations satisfying $f_{1}(t, 0)=0, f_{2}(t, 0)=0$ and

$$
\begin{gathered}
f_{1}^{T}(t, x(t)) f_{1}(t, x(t)) \leq \alpha^{2} x^{T}(t) x(t) \\
f_{2}^{T}(t, x(t-\tau(t))) f_{2}(t, x(t-\tau(t))) \leq \beta^{2} x^{T}(t-\tau(t)) x(t-\tau(t)),
\end{gathered}
$$

where $\alpha$ and $\beta$ are positive real numbers.

The uncertain matrices $A(t), B(t), D_{1}(t)$, and $D_{2}(t)$ satisfy

$$
\begin{array}{cc}
A(t)=A+\Delta A(t), & B(t)=B+\Delta B(t), \\
D_{1}(t)=D_{1}+\Delta D_{1}(t), & D_{2}(t)=D_{2}+\Delta D_{2}(t),
\end{array}
$$

where $A, B, D_{1}, D_{2} \in \mathbb{R}^{n \times n}$ are constant matrices with appropriate dimension, and $\Delta A(t)$, $\Delta B(t), \Delta D_{1}(t)$, and $\Delta D_{2}(t)$ are unknown real matrices of appropriate dimension representing the systems time-varying parameter uncertainties which satisfy

$$
\begin{array}{cc}
\Delta A(t)=G_{1} F(t) E_{A}, & \Delta B(t)=G_{2} F(t) E_{B}, \\
\Delta D_{1}(t)=G_{3} F(t) E_{D_{1}}, & \Delta D_{2}(t)=G_{4} F(t) E_{D_{2}},
\end{array}
$$

where $G_{1}, G_{2}, G_{3}, G_{4}, E_{A}, E_{B}, E_{D_{1}}$, and $E_{D_{2}}$ are known real constant matrices of appropriate dimension. $F(t)$ is unknown time-varying matrix satisfying

$$
F^{T}(t) F(t) \leq I
$$

For simplicity, we denote $f_{1}(t, x(t)), f_{2}(t, x(t-\tau(t)))$, by $f_{1}, f_{2}$, respectively.

Let $\tau_{e}=(1 / 2)\left(\tau_{M}+\tau_{m}\right)$ and $\rho=(1 / 2)\left(\tau_{M}-\tau_{m}\right)$. Then $\tau(t)$ can be expressed as

$$
\tau(t)=\tau_{e}+\rho \xi(t),
$$

where

$$
\xi(t)= \begin{cases}\frac{2 \tau(t)-\left(\tau_{M}+\tau_{m}\right)}{\tau_{M}-\tau_{m}}, & \tau_{M}>\tau_{m} \\ 0, & \tau_{M}=\tau_{m} .\end{cases}
$$


Obviously, $|\xi(t)| \leq 1$. For this case, $\tau(t)$ is a function belonging to the interval $\left[\tau_{e}-\rho, \tau_{e}+\rho\right]$, where $\rho$ can be taken as the range of variation of the time-varying delay $\tau(t)$. Using the fact that

$$
x\left(t-\tau_{e}\right)-x(t-\tau(t))=\int_{t-\tau(t)}^{t-\tau_{e}} \dot{x}(s) d s
$$

system (2.1) can be rewritten as

$$
\begin{aligned}
\dot{x}(t)-C \dot{x}(t-d(t))= & A(t) x(t)+B(t) x\left(t-\tau_{e}\right)-B(t) \int_{t-\tau(t)}^{t-\tau_{e}} \dot{x}(s) d s \\
& +D_{1}(t) f_{1}+D_{2}(t) f_{2} .
\end{aligned}
$$

Lemma 2.1 (see [17]). There exists a symmetric matrix $X$ such that

$$
\left[\begin{array}{cc}
P_{1}-L X L^{T} & Q_{1} \\
Q_{1}^{T} & R_{1}
\end{array}\right]<0, \quad\left[\begin{array}{cc}
P_{2}+X & Q_{2} \\
Q_{2}^{T} & R_{2}
\end{array}\right]<0
$$

if and only if

$$
\left[\begin{array}{ccc}
P_{1}+L P_{2} L^{T} & Q_{1} & L Q_{2} \\
Q_{1}^{T} & R_{1} & 0 \\
Q_{2}^{T} L^{T} & 0 & R_{2}
\end{array}\right]<0
$$

Lemma 2.2 (see [3]). For any constant symmetric matrix $M \in R^{n \times n}, M=M^{T}>0,0 \leq h_{m} \leq$ $h(t) \leq h_{M}, t \geq 0$, and any differentiable vector function $x(t) \in R^{n}$, we have

$$
\begin{aligned}
\text { (a) }\left[\int_{t-h_{m}}^{t} \dot{x}(s) d s\right]^{T} M\left[\int_{t-h_{m}}^{t} \dot{x}(s) d s\right] & \leq h_{m} \int_{t-h_{m}}^{t} \dot{x}^{T}(s) M \dot{x}(s) d s, \\
\text { (b) }\left[\int_{t-h(t)}^{t-h_{m}} \dot{x}(s) d s\right]^{T} M\left[\int_{t-h(t)}^{t-h_{m}} \dot{x}(s) d s\right] & \leq\left(h(t)-h_{m}\right) \int_{t-h(t)}^{t-h_{m}} \dot{x}^{T}(s) M \dot{x}(s) d s \\
& \leq\left(h_{M}-h_{m}\right) \int_{t-h(t)}^{t-h_{m}} \dot{x}^{T}(s) M \dot{x}(s) d s .
\end{aligned}
$$

Lemma 2.3 (see [19]). Given matrices $Q=Q^{T}, H, E$, and $R=R^{T}>0$ with appropriate dimensions. Then

$$
Q+H F E+E^{T} F^{T} H^{T}<0
$$


Journal of Applied Mathematics

for all $F$ satisfying $F^{T} F \leq R$, if and only if there exists an $\epsilon>0$ such that

$$
Q+\epsilon H H^{T}+\epsilon^{-1} E^{T} R E<0 .
$$

Proposition 2.4 (Cauchy inequality). For any symmetric positive definite matrix $N \in M^{n \times n}$ and $x, y \in \mathbb{R}^{n}$, we have

$$
\pm 2 x^{T} y \leq x^{T} N x+y^{T} N^{-1} y
$$

\section{Main Results}

Now we present a new delay-dependent condition for the asymptotic stability of system (2.1).

Assumption 3.1. All the eigenvalues of matrix $\mathrm{C}$ are inside the unit circle.

First, we study the problem of stability for nominal system of (2.10) with $\Delta A(t)=0$, $\Delta B(t)=0, \Delta D_{1}(t)=0$, and $\Delta D_{2}(t)=0$.

Theorem 3.2. Under Assumption 3.1, nominal system of (2.10) with time-varying delay satisfying (2.2) is asymptotically stable if there exist positive definite matrices $P, Q, Q_{1}, R, S, W$, matrices $K_{1}$, $K_{2}, L_{i}, M_{i}, i=1,2, \ldots, 7$ of appropriate dimension and $\delta_{1}, \delta_{2}>0$ such that

$$
\Sigma_{1}=\left[\begin{array}{ccccccccc}
\phi_{11} & \phi_{12} & \phi_{13} & \phi_{14} & \phi_{15} & \phi_{16} & \phi_{17} & \tau_{e} L_{1}^{T} & \rho M_{1} \\
* & \phi_{22} & \phi_{23} & \phi_{24} & \phi_{25} & \phi_{26} & \phi_{27} & \tau_{e} L_{2}^{T} & \rho M_{2} \\
* & * & \phi_{33} & \phi_{34} & \phi_{35} & \phi_{36} & \phi_{37} & \tau_{e} L_{3}^{T} & \rho M_{3} \\
* & * & * & \phi_{44} & \phi_{45} & \phi_{46} & \phi_{47} & \tau_{e} L_{4}^{T} & \rho\left(K_{1}^{T} B+M_{4}\right) \\
* & * & * & * & \phi_{55} & \phi_{56} & \phi_{57} & \tau_{e} L_{5}^{T} & \rho\left(K_{2}^{T} B+M_{5}\right) \\
* & * & * & * & * & \phi_{66} & 0 & \tau_{e} L_{6}^{T} & \rho M_{6} \\
* & * & * & * & * & * & \phi_{77} & \tau_{e} L_{7}^{T} & \rho M_{7} \\
* & * & * & * & * & * & * & -\tau_{e} R & 0 \\
* & * & * & * & * & * & * & * & -\rho S
\end{array}\right]<0,
$$

where

$$
\begin{gathered}
\phi_{11}=Q+L_{1}+L_{1}^{T}+\varepsilon_{1} \alpha^{2} I, \\
\phi_{12}=M_{1}^{T}+L_{2}, \\
\phi_{13}=-L_{1}^{T}+L_{3}+M_{1}, \\
\phi_{14}=P+A^{T} K_{1}+L_{4}, \\
\phi_{15}=A^{T} K_{2}+L_{5}, \\
\phi_{16}=L_{6}, \\
\phi_{17}=L_{7},
\end{gathered}
$$




$$
\begin{gathered}
\phi_{22}=M_{2}^{T}+M_{2}+\varepsilon_{2} \beta^{2} I-W, \\
\phi_{23}=-L_{2}^{T}-M_{2}^{T}+M_{3}+W, \\
\phi_{24}=M_{4}, \\
\phi_{25}=M_{5}, \\
\phi_{26}=M_{6}, \\
\phi_{27}=M_{7}, \\
\phi_{33}=-Q-L_{3}^{T}-L_{3}-M_{3}^{T}-M_{3}-W, \\
\phi_{34}=B^{T} K_{1}-L_{4}-M_{4}, \\
\phi_{35}=B^{T} K_{2}-L_{5}-M_{5}, \\
\phi_{36}=-L_{6}-M_{6}, \\
\phi_{37}=-L_{7}-M_{7}, \\
\phi_{44}=Q_{1}+\tau_{e} R+\rho S-K_{1}^{T}-K_{1}+\rho^{2} W, \\
\phi_{45}=K_{1}^{T} C-K_{2}, \\
\phi_{46}=K_{1}^{T} D_{1}, \\
\phi_{47}=K_{1}^{T} D_{2}, \\
\phi_{55}=-(1-\delta) Q_{1}+K_{2}^{T} C+C^{T} K_{2}, \\
\phi_{56}=K_{2}^{T} D_{1}, \\
\phi_{57}=K_{2}^{T} D_{2}, \\
\phi_{17}=-\delta_{2} I
\end{gathered}
$$

Proof. We prove that Theorem 3.2 is true for three cases, namely, $\tau_{m} \leq \tau(t)<\tau_{e} ; \tau(t)=\tau_{e}$; $\tau_{e}<\tau(t) \leq \tau_{M}$.

Case $1\left(\tau_{m} \leq \tau(t)<\tau_{e}\right)$. Choose a Lyapunov-Krasovskii functional candidate as

$$
\begin{aligned}
V_{1}\left(x_{t}\right)= & x^{T}(t) P x(t)+\int_{t-\tau_{e}}^{t} x^{T}(s) Q x(s) d s \\
& +\int_{t-d(t)}^{t} \dot{x}^{T}(s) Q_{1} \dot{x}(s) d s \int_{-\tau_{e}}^{0} \int_{t+s}^{t} \dot{x}^{T}(\theta) R \dot{x}(\theta) d \theta d s \\
& +\int_{-\tau_{e}}^{-\tau_{m}} \int_{t+s}^{t} \dot{x}^{T}(\theta) S \dot{x}(\theta) d \theta d s+\rho \int_{-\tau_{e}}^{-\tau_{m}} \int_{t+s}^{t} \dot{x}^{T}(\theta) W \dot{x}(\theta) d \theta d s,
\end{aligned}
$$


where $P, Q, Q_{1}, R, S$, and $W$ are positive definite matrices. Taking the derivative of $V_{1}\left(x_{t}\right)$ with respect to $t$ along the trajectory of (2.10) yields

$$
\begin{aligned}
\dot{V}_{1}\left(x_{t}\right)= & 2 x^{T}(t) P \dot{x}(t)+x^{T}(t) Q x(t)-x^{T}\left(t-\tau_{e}\right) Q x\left(t-\tau_{e}\right) \\
& +\dot{x}^{T}(t) Q_{1} \dot{x}(t)-(1-\dot{d}(t)) \dot{x}^{T}(t-d(t)) Q_{1} \dot{x}^{T}(t-d(t)) \\
& +\dot{x}^{T}(t)\left(\tau_{e} R+\rho^{2} W+\rho S\right) \dot{x}(t) \\
& -\int_{t-\tau_{e}}^{t} \dot{x}^{T}(s) R \dot{x}(s) d s-\int_{t-\tau_{e}}^{t-\tau_{m}} \dot{x}^{T}(s) S \dot{x}(s) d s \\
& -\rho \int_{t-\tau_{e}}^{t-\tau_{m}} \dot{x}^{T}(s) W \dot{x}(s) d s \\
\leq & 2 x^{T}(t) P \dot{x}^{\prime}(t)+x^{T}(t) Q x(t)-x^{T}\left(t-\tau_{e}\right) Q x\left(t-\tau_{e}\right) \\
& +\dot{x}^{T}(t) Q_{1} \dot{x}(t)-(1-\delta) \dot{x}^{T}(t-d(t)) Q_{1} \dot{x}^{T}(t-d(t)) \\
& +\dot{x}^{T}(t)\left(\tau_{e} R+\rho^{2} W+\rho S\right) \dot{x}(t) \\
& -\int_{t-\tau_{e}}^{t} \dot{x}^{T}(s) R \dot{x}(s) d s-\int_{t-\tau_{e}}^{t-\tau_{m}} \dot{x}^{T}(s) S \dot{x}(s) d s \\
& -\rho \int_{t-\tau_{e}}^{t-\tau_{m}} \dot{x}^{T}(s) W \dot{x}(s) d s,
\end{aligned}
$$

since

$$
\begin{gathered}
-\int_{t-\tau_{e}}^{t-\tau_{m}} \dot{x}^{T}(s) S \dot{x}(s) d s \leq-\int_{t-\tau_{e}}^{t-\tau(t)} \dot{x}^{T}(s) S \dot{x}(s) d s, \\
-\rho \int_{t-\tau_{e}}^{t-\tau_{m}} \dot{x}^{T}(s) W \dot{x}(s) d s \leq-\rho \int_{t-\tau_{e}}^{t-\tau(t)} \dot{x}^{T}(s) W \dot{x}(s) d s .
\end{gathered}
$$

Based on Lemma 2.2, we obtain

$$
\begin{aligned}
-\rho \int_{t-\tau_{e}}^{t-\tau(t)} \dot{x}^{T}(s) W \dot{x}(s) d s \leq & -\left(\tau_{e}-\tau(t)\right) \int_{t-\tau_{e}}^{t-\tau(t)} \dot{x}^{T}(s) W \dot{x}(s) d s \\
\leq & -x^{T}\left(t-\tau_{e}\right) W x\left(t-\tau_{e}\right)+2 x^{T}\left(t-\tau_{e}\right) W x(t-\tau(t)) \\
& -x^{T}(t-\tau(t)) W x(t-\tau(t))
\end{aligned}
$$


and from the following equalities:

$$
\begin{gathered}
2\left[\dot{x}^{T}(t) K_{1}^{T}+\dot{x}^{T}(t-d(t)) K_{2}^{T}\right] \times\left[A x(t)+B x\left(t-\tau_{e}\right)-B \int_{t-\tau(t)}^{t-\tau_{e}} \dot{x}(s) d s\right. \\
\left.+D_{1} f_{1}+D_{2} f_{2}+C \dot{x}(t-d(t))-\dot{x}(t)\right]=0, \\
2\left[x^{T}(t) L_{1}^{T}+x^{T}(t-\tau(t)) L_{2}^{T}+x^{T}\left(t-\tau_{e}\right) L_{3}^{T}+\dot{x}^{T}(t) L_{4}^{T}+\dot{x}^{T}(t-d(t)) L_{5}^{T}\right. \\
\left.+f_{1}^{T} L_{6}^{T}+f_{2}^{T} L_{7}^{T}\right] \times\left[x(t)-x\left(t-\tau_{e}\right)-\int_{t-\tau_{e}}^{t} \dot{x}(s) d s\right]=0, \\
2\left[x^{T}(t) M_{1}^{T}+x^{T}(t-\tau(t)) M_{2}^{T}+x^{T}\left(t-\tau_{e}\right) M_{3}^{T}+\dot{x}^{T}(t) M_{4}^{T}+\dot{x}^{T}(t-d(t)) M_{5}^{T}\right. \\
\left.+f_{1}^{T} M_{6}^{T}+f_{2}^{T} M_{7}^{T}\right] \times\left[x(t-\tau(t))-x\left(t-\tau_{e}\right)-\int_{t-\tau_{e}}^{t-\tau(t)} \dot{x}(s) d s\right]=0,
\end{gathered}
$$

where $K_{1}, K_{2}$, and $L_{i}, M_{i}, i=1,2, \ldots, 7$ are some matrices of appropriate dimension. Next, from (4.5), for any scalars $\delta_{1}>0$ and $\delta_{2}>0$, we obtain

$$
\begin{gathered}
\delta_{1}\left[\alpha^{2} x^{T}(t) x(t)-f_{1}^{T} f_{1}\right] \geq 0, \\
\delta_{2}\left[\beta^{2} x^{T}(t-\tau(t)) x(t-\tau(t))-f_{2}^{T} f_{2}\right] \geq 0 .
\end{gathered}
$$

By adding the terms on left of (3.7)-(3.10) to $\dot{V}_{1}\left(x_{t}\right)$, we may express $\dot{V}_{1}\left(x_{t}\right)$ as

$$
\begin{aligned}
\dot{V}_{1}\left(x_{t}\right) \leq & 2 x^{T}(t) P \dot{x}(t)+x^{T}(t) Q x(t)-x^{T}\left(t-\tau_{e}\right) Q x\left(t-\tau_{e}\right)+\dot{x}^{T}(t) Q_{1} \dot{x}(t) \\
- & (1-\delta) \dot{x}^{T}(t-d(t)) Q_{1} \dot{x}(t-d(t))+\dot{x}^{T}(t)\left(\tau_{e} R+\rho S+\rho^{2} W\right) \dot{x}(t) \\
- & \int_{t-\tau_{e}}^{t} \dot{x}^{T}(s) R \dot{x}(s) d s-\int_{t-\tau_{e}}^{t-\tau(t)} \dot{x}^{T}(s) S \dot{x}(s) d s+2\left[\dot{x}^{T}(t) K_{1}^{T}+\dot{x}^{T}(t-d(t)) K_{2}^{T}\right] \\
\times & {\left[A x(t)+B x\left(t-\tau_{e}\right)+B \int_{t-\tau_{e}}^{t-\tau(t)} \dot{x}(s) d s+D_{1} f_{1}+D_{2} f_{2}+C \dot{x}(t-d(t))-\dot{x}(t)\right] } \\
+ & 2\left[x^{T}(t) L_{1}^{T}+x^{T}(t-\tau(t)) L_{2}^{T}+x^{T}\left(t-\tau_{e}\right) L_{3}^{T}+\dot{x}^{T}(t) L_{4}^{T}+\dot{x}^{T}(t-d(t)) L_{5}^{T}\right. \\
& \left.\quad+f_{1}^{T} L_{6}^{T}+f_{2}^{T} L_{7}^{T}\right] \times\left[x(t)-x\left(t-\tau_{e}\right)-\int_{t-\tau_{e}}^{t} \dot{x}^{(s)} d s\right] \\
+ & 2\left[x^{T}(t) M_{1}^{T}+x^{T}(t-\tau(t)) M_{2}^{T}\right. \\
& \left.\quad+x^{T}\left(t-\tau_{e}\right) M_{3}^{T}+\dot{x}^{T}(t) M_{4}^{T}+\dot{x}^{T}(t-d(t)) M_{5}^{T}+f_{1}^{T} M_{6}^{T}+f_{2}^{T} M_{7}^{T}\right] \\
\times & {\left[x(t-\tau(t))-x\left(t-\tau_{e}\right)-\int_{t-\tau_{e}}^{t-\tau(t)} \dot{x}(s) d s\right]+\delta_{1}\left[\alpha^{2} x^{T}(t) x(t)-f_{1}^{T} f_{1}\right] }
\end{aligned}
$$


Journal of Applied Mathematics

$$
\begin{aligned}
& -x^{T}\left(t-\tau_{e}\right) W x\left(t-\tau_{e}\right)+2 x^{T}\left(t-\tau_{e}\right) W x(t-\tau(t)) \\
& -x^{T}(t-\tau(t)) W x(t-\tau(t))+\delta_{2}\left[\beta^{2} x^{T}(t-\tau(t)) x(t-\tau(t))-f_{2}^{T} f_{2}\right] \\
= & \frac{1}{\tau_{e}} \int_{t-\tau_{e}}^{t} \omega^{T}(t, s) \phi_{1} \omega(t, s) d s+\frac{1}{\tau_{e}-\tau(t)} \int_{t-\tau_{e}}^{t-\tau(t)} \omega^{T}(t, s) \phi_{2} \omega(t, s) d s,
\end{aligned}
$$

where

$$
\omega^{T}(t, s)=\left[x^{T}(t) x^{T}(t-\tau(t)) x^{T}\left(t-\tau_{e}\right) \dot{x}^{T}(t) \dot{x}^{T}(t-d(t)) f_{1}^{T} f_{2}^{T}-\dot{x}^{T}(s)\right],
$$

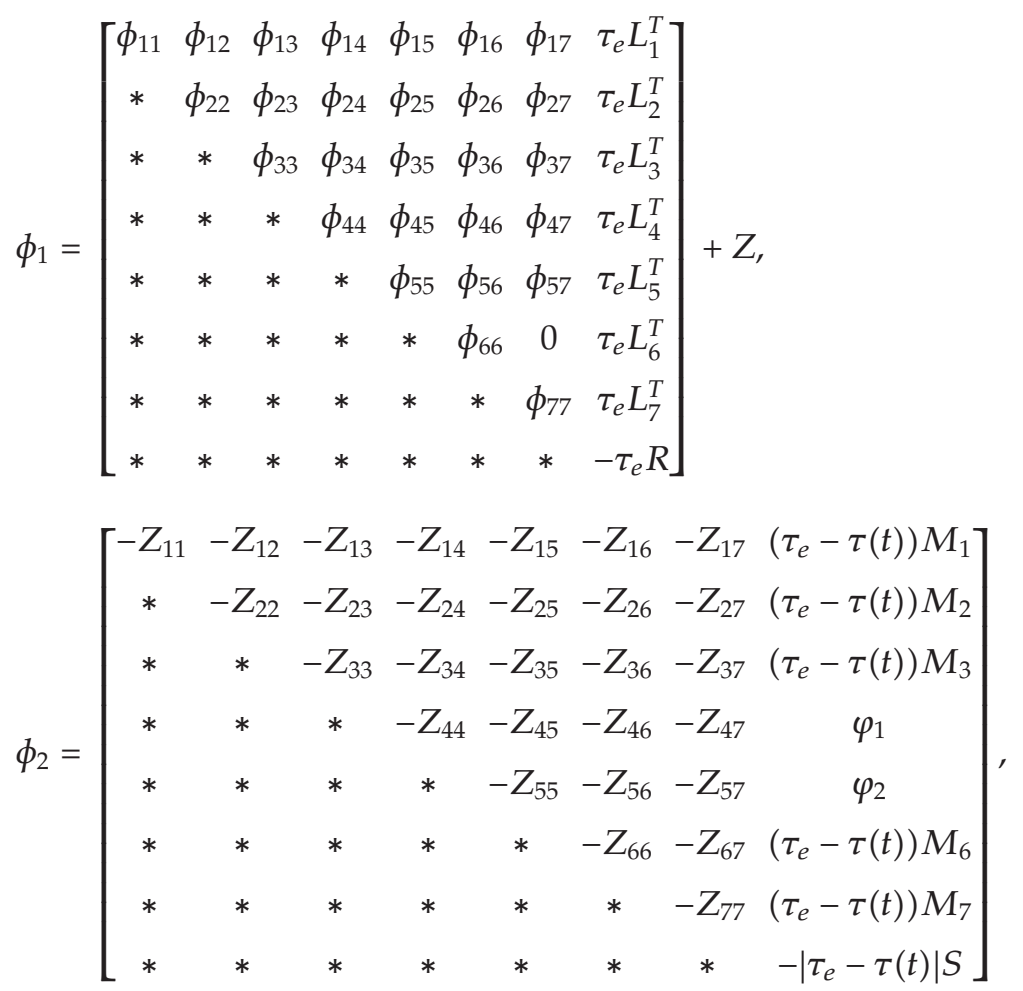$$
Z=\left[\begin{array}{cccccccc}
Z_{11} & Z_{12} & Z_{13} & Z_{14} & Z_{15} & Z_{16} & Z_{17} & 0 \\
* & Z_{22} & Z_{23} & Z_{24} & Z_{25} & Z_{26} & Z_{27} & 0 \\
* & * & Z_{33} & Z_{34} & Z_{35} & Z_{36} & Z_{37} & 0 \\
* & * & * & Z_{44} & Z_{45} & Z_{46} & Z_{47} & 0 \\
* & * & * & * & Z_{55} & Z_{56} & Z_{57} & 0 \\
* & * & * & * & * & Z_{66} & Z_{67} & 0 \\
* & * & * & * & * & * & Z_{77} & 0 \\
* & * & * & * & * & * & * & 0
\end{array}\right],
$$ 
$\varphi_{i}=\left(\tau_{e}-\tau(t)\right)\left(-K_{i}^{T} B+M_{i}\right), i=1,2, Z_{i i}>0, i=1,2, \ldots, 7, Z_{i j}, i=1,2, \ldots, 7, j=i+1, \ldots, 7$ are some parameter matrices of appropriate dimensions. From (3.11) if $\phi_{1}<0$ and $\phi_{2}<0$, then $\dot{V}_{1}\left(x_{t}\right) \leq-\lambda_{1}\|x(t)\|^{2}$ for some $\lambda_{1}>0$. Pre and postmultiplying both sides of $\phi_{2}<0$ by $\operatorname{diag}\left\{I, I, I, I, I, I, I, \operatorname{sgn}\left(\tau(t)-\tau_{e}\right)\right\}$, we get that

$$
\phi_{2} \leq\left[\begin{array}{cccccccc}
-Z_{11} & -Z_{12} & -Z_{13} & -Z_{14} & -Z_{15} & -Z_{16} & -Z_{17} & \rho M_{1} \\
* & -Z_{22} & -Z_{23} & -Z_{24} & -Z_{25} & -Z_{26} & -Z_{27} & \rho M_{2} \\
* & * & -Z_{33} & -Z_{34} & -Z_{35} & -Z_{36} & -Z_{37} & \rho M_{3} \\
* & * & * & -Z_{44} & -Z_{45} & -Z_{46} & -Z_{47} & \rho\left(-K_{1}^{T} B+M_{4}\right) \\
* & * & * & * & -Z_{55} & -Z_{56} & -Z_{57} & \rho\left(-K_{2}^{T} B+M_{5}\right) \\
* & * & * & * & * & -Z_{66} & -Z_{67} & \rho M_{6} \\
* & * & * & * & * & * & -Z_{77} & \rho M_{7} \\
* & * & * & * & * & * & * & -\rho S
\end{array}\right]<0 .
$$

By Schur complement lemma, this implies $\phi_{2}<0$. In light of Lemma 2.1, (3.1) holds if and only if $\phi_{1}<0$ and (3.13) simultaneously hold. Then (3.1) holds if and only if there exists a symmetric matrix $Z, \phi_{1}<0$ and (3.13) simultaneously hold. Therefore, nominal system of (2.10) is asymptotically stable.

Case $2\left(\tau(t)=\tau_{e}\right)$. For this case, we choose a Lyapunov-Krasovskii functional candidate as

$$
\begin{aligned}
V_{2}\left(x_{t}\right)= & x^{T}(t) P x(t)+\int_{t-\tau_{e}}^{t} x^{T}(s) Q x(s) d s+\int_{t-d(t)}^{t} \dot{x}^{T}(s) Q_{1} \dot{x}(s) d s \\
& +\int_{-\tau_{e}}^{0} d s \int_{t+s}^{t} \dot{x}^{T}(\theta) R \dot{x}(\theta) d \theta,
\end{aligned}
$$

where $P, Q, Q_{1}$, and $R$ positive definite matrices are the same as those in $V_{1}\left(x_{t}\right)$.

Case $3\left(\tau_{e}<\tau(t)<\tau_{M}\right)$. For this case, we choose the Lyapunov-Krasovskii functional candidate as

$$
\begin{aligned}
V_{3}\left(x_{t}\right)= & x^{T}(t) P x(t)+\int_{t-\tau_{e}}^{t} x^{T}(s) Q x(s) d s \\
& +\int_{t-d(t)}^{t} \dot{x}^{T}(s) Q_{1} \dot{x}(s) d s \int_{-\tau_{e}}^{0} \int_{t+s}^{t} \dot{x}^{T}(\theta) R \dot{x}(\theta) d \theta d s \\
& +\int_{-\tau_{M}}^{-\tau_{e}} \int_{t+s}^{t} \dot{x}^{T}(\theta) S \dot{x}(\theta) d \theta d s+\rho \int_{-\tau_{M}}^{-\tau_{e}} \int_{t+s}^{t} \dot{x}^{T}(\theta) W \dot{x}(\theta) d \theta d s,
\end{aligned}
$$

where $P, Q, Q_{1}, R, S$, and $W$ are positive definite matrices and are the same as those in $V_{1}\left(x_{t}\right)$.

By similar arguments used in proof of Theorem 3.2, we conclude that the nominal system of (2.10) is robustly asymptotically stable. The proof is complete. 
Journal of Applied Mathematics

Based on Theorem 3.2, we can perform the robust stability analysis for system (2.10) with uncertainties (2.5) and (2.6).

Theorem 3.3. Under Assumption 3.1, system (2.10) with time-varying delay satisfying (2.2) and uncertainties (2.5) and (2.6) is asymptotically stable if there exist positive definite matrices $P, Q, Q_{1}$, $R, S, W$ and $K_{1}, K_{2}, L_{i}, M_{i}, i=1,2, \ldots, 7$ of appropriate dimension and scalars $\epsilon_{i}>0, i=1,2, \ldots, 10$ such that

$$
\begin{aligned}
& \mathcal{M}=\left[\begin{array}{ccccccccc}
M_{11} & \phi_{12} & \phi_{13} & \phi_{14} & \phi_{15} & \phi_{16} & \phi_{17} & \tau_{e} L_{1}^{T} & \rho M_{1} \\
* & \phi_{22} & \phi_{23} & \phi_{24} & \phi_{25} & \phi_{26} & \phi_{27} & \tau_{e} L_{2}^{T} & \rho M_{2} \\
* & * & M_{33} & \phi_{34} & \phi_{35} & \phi_{36} & \phi_{37} & \tau_{e} L_{3}^{T} & \rho M_{3} \\
* & * & * & M_{44} & \phi_{45} & \phi_{46} & \phi_{47} & \tau_{e} L_{4}^{T} & \rho\left(K_{1}^{T} B+M_{4}\right) \\
* & * & * & * & M_{55} & \phi_{56} & \phi_{57} & \tau_{e} L_{5}^{T} & \rho\left(K_{2}^{T} B+M_{5}\right) \\
* & * & * & * & * & M_{66} & 0 & \tau_{e} L_{6}^{T} & \rho M_{6} \\
* & * & * & * & * & * & M_{77} & \tau_{e} L_{7}^{T} & \rho M_{7} \\
* & * & * & * & * & * & * & -\tau_{e} R & 0 \\
* & * & * & * & * & * & * & * & M_{99}
\end{array}\right]<0, \\
& \mathcal{M}_{1}=\left[\begin{array}{cccccc}
-0.1 K_{1}^{T}-0.1 K_{1} & K_{1}^{T} G_{1} & K_{1}^{T} G_{2} & K_{1}^{T} G_{2} & K_{1}^{T} G_{3} & K_{1}^{T} G_{4} \\
G_{1}^{T} K_{1} & -\epsilon_{1} I & 0 & 0 & 0 & 0 \\
G_{2}^{T} K_{1} & 0 & -\epsilon_{2} I & 0 & 0 & 0 \\
G_{2}^{T} K_{1} & 0 & 0 & -\epsilon_{3} I & 0 & 0 \\
G_{3}^{T} K_{1} & 0 & 0 & 0 & -\epsilon_{4} I & 0 \\
G_{4}^{T} K_{1} & 0 & 0 & 0 & 0 & -\epsilon_{5} I
\end{array}\right]<0, \\
& \mathcal{M}_{2}=\left[\begin{array}{cccccc}
-0.1 Q_{1}+0.1 K_{2}^{T} C+0.1 C^{T} K_{2} & K_{2}^{T} G_{1} & K_{2}^{T} G_{2} & K_{2}^{T} G_{2} & K_{2}^{T} G_{3} & K_{2}^{T} G_{4} \\
G_{1}^{T} K_{2} & -\epsilon_{6} I & 0 & 0 & 0 & 0 \\
G_{2}^{T} K_{2} & 0 & -\epsilon_{7} I & 0 & 0 & 0 \\
G_{2}^{T} K_{2} & 0 & 0 & -\epsilon_{8} I & 0 & 0 \\
G_{3}^{T} K_{2} & 0 & 0 & 0 & -\epsilon_{9} I & 0 \\
G_{4}^{T} K_{2} & 0 & 0 & 0 & 0 & -\epsilon_{10} I
\end{array}\right]<0,
\end{aligned}
$$

where

$$
\begin{aligned}
& M_{11}=\phi_{11}+\epsilon_{1} E_{A}^{T} E_{A}+\epsilon_{6} E_{A}^{T} E_{A}, \\
& M_{33}=\phi_{33}+\epsilon_{2} E_{B}^{T} E_{B}+\epsilon_{7} E_{B}^{T} E_{B}, \\
& M_{44}=Q_{1}+\tau_{e} R+\rho S-0.9 K_{1}^{T}-0.9 K_{1}+\rho^{2} W, \\
& M_{55}=-0.9 Q_{1}+\delta Q_{1}+0.9 K_{2}^{T} C+0.9 C^{T} K_{2},
\end{aligned}
$$




$$
\begin{aligned}
& M_{66}=\phi_{66}+\epsilon_{4} E_{D_{1}}^{T} E_{D_{1}}+\epsilon_{9} \rho^{2} E_{B}^{T} E_{B}, \\
& M_{77}=\phi_{77}+\epsilon_{5} E_{D_{2}}^{T} E_{D_{2}}+\epsilon_{10} \rho^{2} E_{B}^{T} E_{B}, \\
& M_{99}=-\rho S+\epsilon_{3} \rho^{2} E_{B}^{T} E_{B}+\epsilon_{8} \rho^{2} E_{B}^{T} E_{B} .
\end{aligned}
$$

Proof. We choose Lyapunov-Krasovskii functional as in Theorem 3.2, we may proof this Theorem by using a similar arguments as in the proof of Theorem 3.2. By replacing $A, B$, $D_{1}$, and $D_{2}$ in (3.11) with $A+G F(t) E_{A}, B+G F(t) E_{B}, D_{1}+G F(t) E_{D_{1}}$ and $D_{2}+G F(t) E_{D_{2}}$, respectively. For Case 1

$$
\begin{aligned}
\dot{V}_{1}\left(x_{t}\right) \leq & 2 x^{T}(t) P \dot{x}(t)+x^{T}(t) Q x(t)-x^{T}\left(t-\tau_{e}\right) Q x\left(t-\tau_{e}\right)+\dot{x}^{T}(t) Q_{1} \dot{x}(t) \\
- & (1-\delta) \dot{x}^{T}(t-d(t)) Q_{1} \dot{x}(t-d(t))+\dot{x}^{T}(t)\left(\tau_{e} R+\rho S+\rho^{2} W\right) \dot{x}(t) \\
- & \int_{t-\tau_{e}}^{t} \dot{x}^{T}(s) R \dot{x}(s) d s-\int_{t-\tau_{e}}^{t-\tau(t)} \dot{x}^{T}(s) S \dot{x}(s) d s+2\left[\dot{x}^{T}(t) K_{1}^{T}+\dot{x}^{T}(t-d(t)) K_{2}^{T}\right] \\
\times & {\left[\left(A+G_{1} F(t) E_{A}\right) x(t)+\left(B+G_{2} F(t) E_{B}\right) x\left(t-\tau_{e}\right)+\left(B+G_{2} F(t) E_{B}\right)\right.} \\
& \left.\times \int_{t-\tau_{e}}^{t-\tau(t)} \dot{x}(s) d s+\left(D_{1}+G_{3} F(t) E_{D_{1}}\right) f_{1}+\left(D_{2}+G_{4} F(t) E_{D_{2}}\right) f_{2}+C \dot{x}(t-d(t))-\dot{x}(t)\right] \\
+ & 2\left[x^{T}(t) L_{1}^{T}+x^{T}(t-\tau(t)) L_{2}^{T}+x^{T}\left(t-\tau_{e}\right) L_{3}^{T}+\dot{x}^{T}(t) L_{4}^{T}+\dot{x}^{T}(t-d(t)) L_{5}^{T}\right. \\
& \left.+f_{1}^{T} L_{6}^{T}+f_{2}^{T} L_{7}^{T}\right] \times\left[x(t)-x\left(t-\tau_{e}\right)-\int_{t-\tau_{e}}^{t} \dot{x}^{(s) d s]}\right. \\
+ & 2\left[x^{T}(t) M_{1}^{T}+x^{T}(t-\tau(t)) M_{2}^{T}+x^{T}\left(t-\tau_{e}\right) M_{3}^{T}+\dot{x}^{T}(t) M_{4}^{T}+\dot{x}^{T}(t-d(t)) M_{5}^{T}\right. \\
& \left.+f_{1}^{T} M_{6}^{T}+f_{2}^{T} M_{7}^{T}\right]\left[x(t-\tau(t))-x\left(t-\tau_{e}\right)-\int_{t-\tau_{e}}^{t-\tau(t)} \dot{x}(s) d s\right]+\delta_{1}\left[\alpha^{2} x^{T}(t) x(t)-f_{1}^{T} f_{1}\right] \\
- & x^{T}\left(t-\tau_{e}\right) W x\left(t-\tau_{e}\right)+2 x^{T}\left(t-\tau_{e}\right) W x(t-\tau(t)) \\
- & x^{T}(t-\tau(t)) W x(t-\tau(t))+\delta_{2}\left[\beta^{2} x^{T}(t-\tau(t)) x(t-\tau(t))-f_{2}^{T} f_{2}\right] .
\end{aligned}
$$

Applying Lemmas 2.3. and 2.4., the following estimations hold:

$$
\begin{aligned}
2 \dot{x}^{T}(t) K_{1}^{T}\left(A+G_{1} F(t) E_{A}\right) x(t) \leq & 2 \dot{x}^{T}(t) K_{1}^{T} A x(t) \\
& +\epsilon_{1}^{-1} \dot{x}^{T}(t) K_{1}^{T} G_{1} G_{1}^{T} K_{1} \dot{x}(t) \\
& +\epsilon_{1} x^{T}(t) E_{A}^{T} E_{A} x(t),
\end{aligned}
$$


Journal of Applied Mathematics

$$
\begin{aligned}
& 2 \dot{x}^{T}(t) K_{1}^{T}\left(B+G_{2} F(t) E_{B}\right) x\left(t-\tau_{e}\right) \leq 2 \dot{x}^{T}(t) K_{1}^{T} B x\left(t-\tau_{e}\right) \\
& +\epsilon_{2}^{-1} \dot{x}^{T}(t) K_{1}^{T} G_{2} G_{2}^{T} K_{1} \dot{x}(t) \\
& +\epsilon_{2} x^{T}\left(t-\tau_{e}\right) E_{B}^{T} E_{B} x\left(t-\tau_{e}\right), \\
& 2 \dot{x}^{T}(t) K_{1}^{T}\left(B+G_{2} F(t) E_{B}\right) \int_{t-\tau_{e}}^{t-\tau(t)} \dot{x}(s) d s \leq 2 \dot{x}^{T}(t) K_{1}^{T} B \int_{t-\tau_{e}}^{t-\tau(t)} \dot{x}(s) d s \\
& +\epsilon_{3}^{-1} \dot{x}^{T}(t) K_{1}^{T} G_{2} G_{2}^{T} K_{4} \dot{x}^{T}(t) \\
& +\epsilon_{3} \rho\left(\int_{t-\tau_{e}}^{t-\tau(t)} \dot{x}(s) E_{B}^{T} E_{B} \dot{x}(s) d s\right), \\
& 2 \dot{x}^{T}(t) K_{1}^{T}\left(D_{1}+G_{3} F(t) E_{D_{1}}\right) f_{1} \leq 2 \dot{x}^{T}(t) K_{1}^{T} D_{1} f_{1} \\
& +\epsilon_{4}^{-1} \dot{x}^{T}(t) K_{1}^{T} G_{3} G_{3}^{T} K_{1} \dot{x}(t) \\
& +\epsilon_{4} f_{1}^{T} E_{D_{1}}^{T} E_{D_{1}} f_{1} \text {, } \\
& 2 \dot{x}^{T}(t) K_{1}^{T}\left(D_{2}+G_{4} F(t) E_{D_{2}}\right) f_{2} \leq 2 \dot{x}^{T}(t) K_{1}^{T} D_{2} f_{2} \\
& +\epsilon_{5}^{-1} \dot{x}^{T}(t) K_{1}^{T} G_{4} G_{4}^{T} K_{1} \dot{x}(t) \\
& +\epsilon_{5} f_{2}^{T} E_{D_{2}}^{T} E_{D_{2}} f_{2} \text {, } \\
& 2 \dot{x}^{T}(t-d(t)) K_{2}^{T}\left(A+G_{1} F(t) E_{A}\right) x(t) \leq 2 \dot{x}^{T}(t-d(t)) K_{2}^{T} A x(t) \\
& +\epsilon_{6}^{-1} \dot{x}^{T}(t-d(t)) K_{2}^{T} G_{1} G_{1}^{T} K_{2} \dot{x}(t-d(t)) \\
& +\epsilon_{6} x^{T}(t-d(t)) E_{A}^{T} E_{A} x(t), \\
& 2 \dot{x}^{T}(t-d(t)) K_{2}^{T}\left(B+G_{2} F(t) E_{B}\right) x\left(t-\tau_{e}\right) \leq 2 \dot{x}^{T}(t-d(t)) K_{1}^{T} B x\left(t-\tau_{e}\right) \\
& +\epsilon_{7}^{-1} \dot{x}^{T}(t-d(t)) K_{1}^{T} G_{2} G_{2}^{T} K_{1} \dot{x}(t-d(t)) \\
& +\epsilon_{7} x^{T}\left(t-\tau_{e}\right) E_{B}^{T} E_{B} x\left(t-\tau_{e}\right), \\
& 2 \dot{x}^{T}(t-d(t)) K_{2}^{T}\left(B+G_{2} F(t) E_{B}\right) \int_{t-\tau_{e}}^{t-\tau(t)} \dot{x}(s) d s \leq 2 \dot{x}^{T}(t-d(t)) K_{2}^{T} B \int_{t-\tau_{e}}^{t-\tau(t)} \dot{x}(s) d s \\
& +\epsilon_{8}^{-1} \dot{x}^{T}(t-d(t)) K_{2}^{T} G_{2} G_{2}^{T} K_{2} \dot{x}^{T}(t-d(t)) \\
& +\epsilon_{8} \rho\left(\int_{t-\tau_{e}}^{t-\tau(t)} \dot{x}(s) E_{B}^{T} E_{B} \dot{x}(s) d s\right),
\end{aligned}
$$




$$
\begin{aligned}
2 \dot{x}^{T}(t-d(t)) K_{2}^{T}\left(D_{1}+G_{3} F(t) E_{D_{1}}\right) f_{1} \leq & 2 \dot{x}^{T}(t-d(t)) K_{2}^{T} D_{1} f_{1} \\
& +\epsilon_{9}^{-1} \dot{x}^{T}(t-d(t)) K_{2}^{T} G_{3} G_{3}^{T} K_{2} \dot{x}(t-d(t)) \\
& +\epsilon_{9} f_{1}^{T} E_{D_{1}}^{T} E_{D_{1}} f_{1}, \\
2 \dot{x}^{T}(t-d(t)) K_{2}^{T}\left(D_{2}+G_{4} F(t) E_{D_{2}}\right) f_{2} \leq & 2 \dot{x}^{T}(t-d(t)) K_{2}^{T} D_{2} f_{2} \\
& +\epsilon_{10}^{-1} \dot{x}^{T}(t-d(t)) K_{2}^{T} G_{4} G_{4}^{T} K_{2} \dot{x}(t-d(t)) \\
& +\epsilon_{10} f_{2}^{T} E_{D_{2}}^{T} E_{D_{2}} f_{2} .
\end{aligned}
$$

Therefore, from (3.18)-(3.28), it follows that

$$
\dot{V}_{1}\left(x_{t}\right) \leq \omega^{T}(t, s) \mathcal{M} \omega(t, s)+\dot{x}^{T}(t) \Omega_{1} \dot{x}(t)+\dot{x}^{T}(t-d(t)) \Omega_{2} \dot{x}(t-d(t)),
$$

where

$$
\begin{aligned}
\Omega_{1}= & -0.1 K_{1}^{T}-0.1 K_{1}+\epsilon_{1}^{-1} K_{1}^{T} G_{1} G_{1}^{T} K_{1}+\epsilon_{2}^{-1} K_{1}^{T} G_{2} G_{2}^{T} K_{1} \\
& +\epsilon_{3}^{-1} K_{1}^{T} G_{2} G_{2}^{T} K_{1}+\epsilon_{4}^{-1} K_{1}^{T} G_{3} G_{3}^{T} K_{1}+\epsilon_{5}^{-1} K_{1}^{T} G_{4} G_{4}^{T} K_{1}, \\
\Omega_{2}= & -0.1 Q_{1}^{T}+0.1 K_{2}^{T} C+0.1 C^{T} K_{2}+\epsilon_{6}^{-1} K_{2}^{T} G_{1} G_{1}^{T} K_{2}+\epsilon_{7}^{-1} K_{2}^{T} G_{2} G_{2}^{T} K_{2} \\
& +\epsilon_{8}^{-1} K_{2}^{T} G_{2} G_{2}^{T} K_{2}+\epsilon_{9}^{-1} K_{2}^{T} G_{3} G_{3}^{T} K_{2}+\epsilon_{10}^{-1} K_{2}^{T} G_{4} G_{4}^{T} K_{2} .
\end{aligned}
$$

Applying Schur complement lemma, the inequalities $\Omega_{1}<0$ and $\Omega_{2}<0$ are equivalent to $\mathcal{M}_{1}<0$ and $\boldsymbol{M}_{2}<0$, respectively. Therefore, system (2.10) is robust asymptotically stable if the condition (3.16) holds.

By using arguments similar to the proof of Case 1 for Case 2 and Case 3, we may conclude that the close-loop system (2.10) is robust asymptotically stable.

Remark 3.4. In this paper, the restriction that the state delay is differentiable is not required, which allows state delay to be fast time varying. Meanwhile, this restriction is required in some existing results, see $[1-5,7,9,11-14,16,18]$.

Remark 3.5. In the proof of Theorem 3.3, we need negative definiteness of matrices $\mathcal{M}, \Omega_{1}$ and $\Omega_{2}$ simultaneously. In order to do so, we need to have certain diagonal terms of matrices $\mathcal{M}$, $\Omega_{1}$ and $\Omega_{2}$ being negative. This leads to the splitting of the term $K_{1}$ as $(0.1+0.9) K_{1}$ which is one possibility to achieve such goal.

\section{Numerical Examples}

In this section, we provide numerical examples to show the effectiveness of our theoretical results. 
Table 1: Comparison of the maximum value $\tau_{M}$ for $\gamma=0.1$.

\begin{tabular}{|c|c|c|}
\hline & \multicolumn{2}{|c|}{$\alpha=0, \beta=0.1$} \\
\hline & $\dot{\tau}(t)=0.5$ & $\begin{array}{l}\text { No restriction on } \\
\dot{\tau}(t)\end{array}$ \\
\hline Zhang et al. [1] & 0.704 & - \\
\hline Shen and Zhong [2] & 1.060 & - \\
\hline \multirow[t]{3}{*}{ Ours } & 一 & 1.0762 \\
\hline & \multicolumn{2}{|c|}{$\alpha=0.1, \beta=0.1$} \\
\hline & $\dot{\tau}(t)=0.5$ & $\begin{array}{c}\text { No restriction on } \\
\dot{\tau}(t)\end{array}$ \\
\hline Zhang et al. [1] & 0.689 & - \\
\hline Shen and Zhong [2] & 1.040 & - \\
\hline Ours & - & 1.0638 \\
\hline
\end{tabular}

Example 4.1. Consider the following uncertain neutral system with time-varying delay and nonlinear uncertainties which is studied in $[1,2]$ :

$$
\begin{aligned}
\dot{x}(t)-C \dot{x}(t-d)= & (A+\Delta A(t)) x(t)+(B+\Delta B(t)) x(t-\tau(t))+\left(D_{1}+\Delta D_{1}(t)\right) f_{1}(t, x(t)) \\
& +\left(D_{2}+\Delta D_{2}(t)\right) f_{2}(t, x(t-\tau(t))),
\end{aligned}
$$

where

$$
\begin{gathered}
A=\left[\begin{array}{cc}
-1.2 & -0.1 \\
-0.1 & -1
\end{array}\right], \quad B=\left[\begin{array}{cc}
-0.6 & 0.7 \\
-1 & -0.8
\end{array}\right], \quad C=\left[\begin{array}{cc}
-0.2 & 0 \\
0.2 & -0.1
\end{array}\right], \\
D_{1}=\left[\begin{array}{cc}
-0.1 & 0 \\
0 & -0.1
\end{array}\right], \quad D_{2}=\left[\begin{array}{cc}
-0.1 & 0 \\
0 & -0.1
\end{array}\right], \\
\Delta A(t)=G F(t) E_{A}, \quad \Delta B(t)=G F(t) E_{B}, \quad \Delta D_{1}(t)=G F(t) E_{D_{1}}, \\
\Delta D_{2}(t)=G F(t) E_{D_{2}}, \quad F^{T}(t) F(t) \leq I, \quad G=r I, \quad E_{A}=I, \\
E_{B}=I, \quad E_{D_{1}}=I, \quad E_{D_{2}}=I .
\end{gathered}
$$

It is assumed that the nonlinear uncertainties satisfy

$$
\left\|f_{1}(t, x(t))\right\| \leq \alpha\|x(t)\|, \quad\left\|f_{2}(t, x(t-\tau(t)))\right\| \leq \beta\|x(t-\tau(t))\|, \quad \alpha>0, \beta>0 .
$$

Applying Theorem 3.3, the maximum allowable value of $\tau_{M}$ is given in Table 1 when $\gamma=0.1$ and in Table 2 for $\gamma=0.5$. The results obtained in [1,2] may not be used for the case when $\tau_{m} \neq 0$. Moreover, the differentiability of the time delay $\tau(t)$ is not required in Theorem 3.3. Tables 1 and 2 show that our results significantly improve the results of $[1,2]$. 
Table 2: Comparison of the maximum value $\tau_{M}$ for $\gamma=0.5$.

\begin{tabular}{|c|c|c|}
\hline & \multicolumn{2}{|c|}{$\alpha=0, \beta=0.1$} \\
\hline & $\dot{\tau}(t)=0.5$ & $\begin{array}{c}\text { No restriction on } \\
\dot{\tau}(t)\end{array}$ \\
\hline Zhang et al. [1] & 0.259 & - \\
\hline Shen and Zhong [2] & 0.450 & - \\
\hline \multirow[t]{3}{*}{ Ours } & 一 & 0.5333 \\
\hline & \multicolumn{2}{|c|}{$\alpha=0.1, \beta=0.1$} \\
\hline & $\dot{\tau}(t)=0.5$ & $\begin{array}{c}\text { No restriction on } \\
\dot{\tau}(t)\end{array}$ \\
\hline Zhang et al. [1] & 0.240 & - \\
\hline Shen and Zhong [2] & 0.429 & - \\
\hline Ours & - & 0.5207 \\
\hline
\end{tabular}

Moreover, it should be pointed out that if we let $\tau_{m}=0.1$ and $\tau_{M}=0.85$, then from Theorem 3.3, the solutions of LMI (3.16) are given as follows:

$$
\begin{aligned}
& P=\left[\begin{array}{ll}
2.4597 & 0.3509 \\
0.3509 & 1.9870
\end{array}\right], \quad Q=\left[\begin{array}{ll}
0.9955 & 0.1967 \\
0.1967 & 1.0236
\end{array}\right], \\
& Q_{1}=\left[\begin{array}{cc}
0.5615 & -0.0545 \\
-0.0545 & 0.2292
\end{array}\right], \quad S=\left[\begin{array}{cc}
1.4211 & 0.1224 \\
0.1224 & 1.1393
\end{array}\right] \text {, } \\
& R=\left[\begin{array}{ll}
1.8164 & 0.2718 \\
0.2718 & 1.7699
\end{array}\right], \quad W=\left[\begin{array}{ll}
0.4131 & 0.0520 \\
0.0520 & 0.3768
\end{array}\right] \text {, } \\
& L_{1}=\left[\begin{array}{ll}
-1.8399 & -0.0430 \\
-0.1215 & -1.9400
\end{array}\right], \quad L_{2}=\left[\begin{array}{cc}
1.5341 & 1.0797 \\
-1.1061 & 1.2226
\end{array}\right] \text {, } \\
& L_{3}=\left[\begin{array}{ll}
-0.1656 & -0.0202 \\
-0.1437 & -0.3157
\end{array}\right], \quad L_{4}=\left[\begin{array}{cc}
-1.3144 & -0.4567 \\
0.1806 & -1.3580
\end{array}\right] \text {, } \\
& L_{5}=\left[\begin{array}{cc}
-0.1514 & -0.0592 \\
-0.0410 & 0.0423
\end{array}\right], \quad L_{6}=\left[\begin{array}{cc}
-0.0047 & 0.0064 \\
-0.0036 & -0.0045
\end{array}\right] \text {, } \\
& L_{7}=\left[\begin{array}{cc}
-0.0047 & 0.0064 \\
-0.0037 & -0.0046
\end{array}\right], \quad K_{1}=\left[\begin{array}{ll}
1.7652 & 0.2207 \\
0.0928 & 1.4950
\end{array}\right] \text {, } \\
& K_{2}=\left[\begin{array}{cc}
-0.2816 & 0.0203 \\
0.0408 & -0.1346
\end{array}\right], \quad M_{1}=\left[\begin{array}{cc}
-1.4428 & -0.0867 \\
-0.0535 & -1.3096
\end{array}\right] \text {, } \\
& M_{2}=\left[\begin{array}{cc}
-1.2780 & -1.0258 \\
1.1266 & -0.9849
\end{array}\right], \quad M_{3}=\left[\begin{array}{cc}
0.2312 & -0.1717 \\
0.0660 & 0.4381
\end{array}\right] \text {, }
\end{aligned}
$$




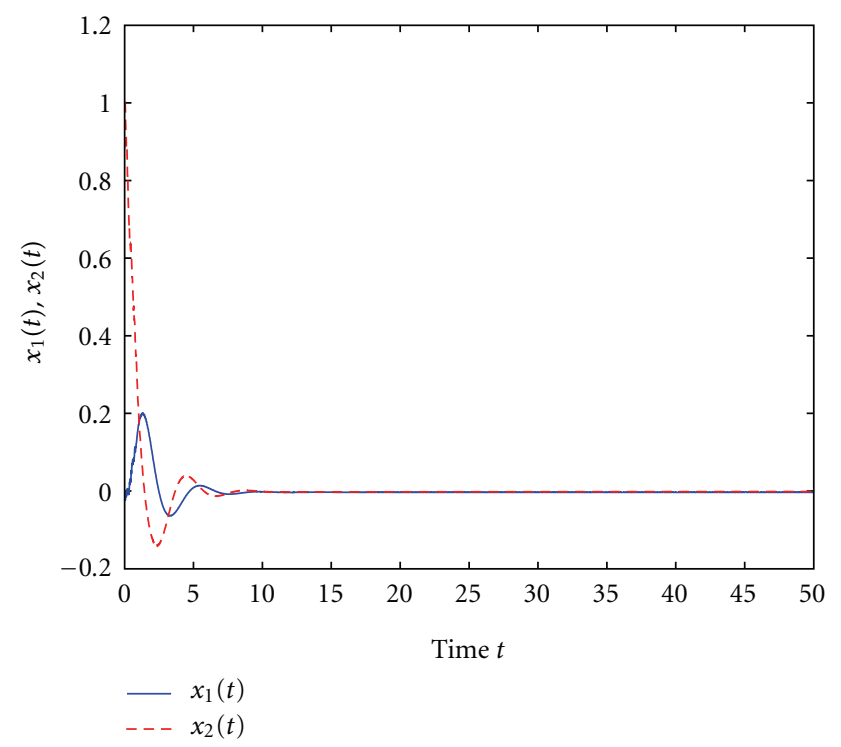

Figure 1: The trajectories of $x_{1}(t)$ and $x_{2}(t)$ of the system (4.1) with time-varying delay $\tau(t)=0.1+$ $0.75|\sin 10 t|$.

$$
\begin{aligned}
M_{4} & =\left[\begin{array}{cc}
0.3926 & -1.0687 \\
0.9108 & 0.5805
\end{array}\right], \quad M_{5}=\left[\begin{array}{cc}
0.3601 & 0.1664 \\
-0.1928 & 0.1095
\end{array}\right], \\
M_{6} & =\left[\begin{array}{cc}
0.0032 & -0.0046 \\
0.0110 & 0.0081
\end{array}\right], \quad M_{7}=\left[\begin{array}{cc}
0.0032 & -0.0047 \\
0.0111 & 0.0081
\end{array}\right], \\
\delta_{1} & =2.0156, \quad \delta_{2}=1.9922, \quad \epsilon_{1}=0.4383 \\
\epsilon_{2} & =0.4197, \quad \epsilon_{3}=0.5513, \quad \epsilon_{4}=0.9140 \\
\epsilon_{5} & =0.9042, \quad \epsilon_{6}=0.3288, \quad \epsilon_{7}=0.3143 \\
\epsilon_{8} & =0.4376, \quad \epsilon_{9}=0.9021, \quad \epsilon_{10}=0.9003 .
\end{aligned}
$$

Figure 1 shows the trajectories of solutions $x_{1}(t)$ and $x_{2}(t)$ of the system (4.1) with time-varying delay $\tau(t)=0.1+0.75|\sin 10 t|, d=1, \phi(t)=[\sin t, \cos t)]$, for all $t \in[-1,0], f_{1}(t, x(t))=\left[0.1 \sin \left|x_{1}(t)\right|, 0.1 \cos \left|x_{2}(t)\right|\right]^{T}, f_{2}(t, x(t-\tau(t)))=\left[0.1 e^{-\sin ^{2} x_{1}(t-\tau(t))}\right.$, $\left.0.1 e^{-\cos ^{2} x_{2}(t-\tau(t))}\right]^{T}$ and $F(t)=\operatorname{diag}\left\{\sin ^{2}(t), \sin ^{2}(t)\right\}$. Since the time-delay $\tau(t)$ is not differentiable, the stability criterion in $[1,2]$ cannot be applied to this case because it is only applicable to the system with the differentiable delay.

Example 4.2. Consider the following uncertain neutral system with time-varying delay in [3, 4]:

$$
\dot{x}(t)-C \dot{x}(t-d(t))=(A+\Delta A(t)) x(t)+(B+\Delta B(t)) x(t-\tau(t)),
$$


Table 3: Maximum allowable upper bounds $\tau_{M}$ of the time-varying delay for different values of the lower bounds $\tau_{m}$ and $c=0.1$.

\begin{tabular}{lcc}
\hline & $\tau_{m}=0$ & $\tau_{m}=0.5$ \\
\hline Yu and Lien [3] & 0.658 & 0.793 \\
Kwon et al. [4] & 0.852 & 0.894 \\
Ours & 0.873 & 0.951 \\
\hline
\end{tabular}

Table 4: Maximum allowable upper bounds $\tau_{M}$ of the time-varying delay for different values of the lower bounds $\tau_{m}, c=0.1$ and $\delta=0.1$.

\begin{tabular}{lcccc}
\hline$\tau_{m}=0$ & $\dot{\tau}(t)=0.5$ & $\dot{\tau}(t)=0.9$ & $\dot{\tau}(t) \geq 1$ & $\begin{array}{c}\text { No restriction } \\
\text { on } \dot{\tau}(t)\end{array}$ \\
\hline Han and Yu [5] & 0.57 & 0.17 & - & - \\
Qiu et al. [6] & 0.7890 & 0.7199 & 0.7216 & - \\
Ours & - & - & - & 0.8676 \\
\hline$\tau_{m}=0.5$ & $\dot{\tau}(t)=0.5$ & $\dot{\tau}(t)=0.9$ & $\dot{\tau}(t) \geq 1$ & $\begin{array}{c}\text { No restriction } \\
\text { on } \dot{\tau}(t)\end{array}$ \\
\hline Ours & - & - & - & 0.9460 \\
\hline
\end{tabular}

where

$$
\begin{gathered}
A=\left[\begin{array}{cc}
-2 & 0 \\
0 & -1
\end{array}\right], \quad B=\left[\begin{array}{cc}
-1 & 0 \\
-1 & -1
\end{array}\right], \quad C=\left[\begin{array}{ll}
c & 0 \\
0 & c
\end{array}\right], \\
\Delta A(t)=\left[\begin{array}{ll}
\gamma_{1} & 0 \\
0 & \gamma_{2}
\end{array}\right], \quad \Delta B(t)=\left[\begin{array}{ll}
\gamma_{3} & 0 \\
0 & \gamma_{4}
\end{array}\right],
\end{gathered}
$$

where $0 \leq|c|<1$, and $\gamma_{i}, i=1,2, \ldots, 4$ are unknown parameter satisfying $\left|\gamma_{1}\right| \leq 1.6,\left|\gamma_{2}\right| \leq 0.05$, $\left|\gamma_{3}\right|<0.1$, and $\left|\gamma_{4}\right|<0.3$.

Case 1. For $c=0.1, \delta=0$, the maximum values of $\tau_{M}$ are listed in Table 3 for $c=0.1$ by applying criteria in $[3,4]$ and in this paper. We see that the maximum allowable bounds for $\tau_{M}$ obtained from Theorem 3.3 are much better than that obtained in $[3,4]$.

Case 2. For $c=0.1, \delta=0.1$, the maximum value $\tau_{M}$ obtained form Theorem 3.3 is listed in Table 4 . In $[3,4]$ the neutral delay is constant, then its stability criterion cannot be applied to systems with time-varying neutral delay. Furthermore, the stability criterion in $[5,6]$ cannot be applied to this case because Theorem 3.3 does not have restriction on the derivative of time-varying delay. It is obvious that the obtained results are significantly better than those in [3-6].

Figure 2 shows the trajectories of solutions $x_{1}(t)$ and $x_{2}(t)$ of the system (4.5) with time-varying delay $\left.\tau(t)=0.3+0.5|\cos 10 t|, d(t)=0.1 \sin ^{2} t, \phi(t)=[\sin t, \cos t)\right]$, for all $t \in$ $[-0.8,0]$. 


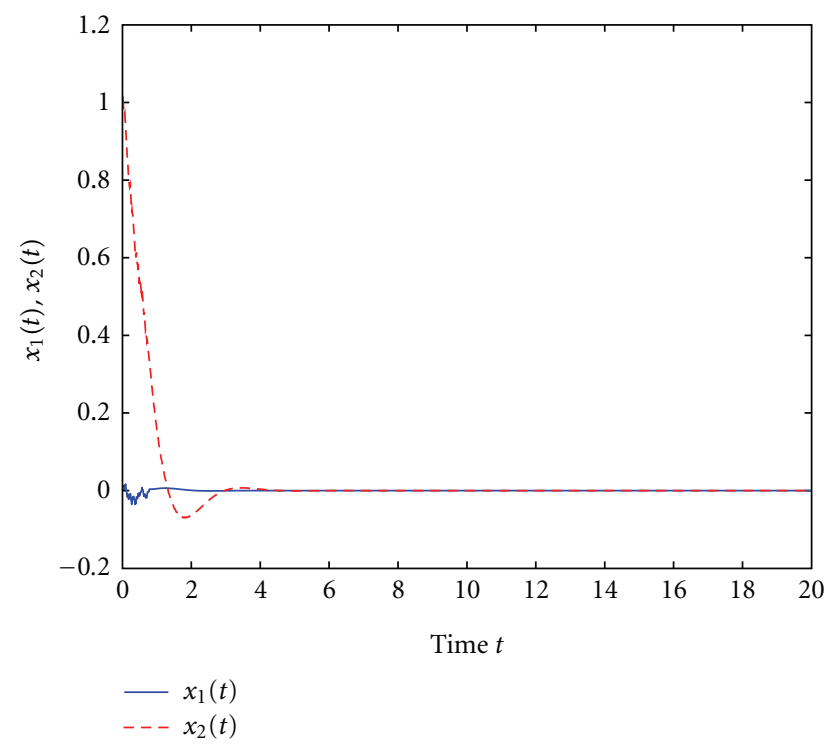

Figure 2: The trajectories of $x_{1}(t)$ and $x_{2}(t)$ of the system (4.5) with time-varying delay $\tau(t)=0.3+$ $0.5|\cos 10 t|$.

\section{Conclusions}

In this paper, we have investigated the delay-dependent robust stability criteria for uncertain neutral systems with interval time-varying delays and time-varying nonlinear perturbations simultaneously. Based on Lyapunov-krasovskii theory, new delay-dependent sufficient conditions for robust stability have been derived in terms of LMIs. The interval time-varying delay function is not required to be differentiable, which allows time-delay function to be a fast time-varying function. Numerical examples are given to illustrate the effectiveness of the theoretic results which show that our results are much less conservative than some existing results in the literature.

\section{Acknowledgments}

Financial support from the Thailand Research Fund through the Royal Golden Jubilee Ph.D. Program (Grant no. PHD/0355/2552) to W. Weera and P. Niamsup is acknowledged. The first author is also supported by the Graduate School, Chiang Mai University, and the Center of Excellence in Mathematics, Thailand. The second author is also supported by the Center of Excellence in Mathematics, Thailand and Commission for Higher Education, Thailand.

\section{References}

[1] J. Zhang, P. Shi, and J. Qiu, “Robust stability criteria for uncertain neutral system with time delay and nonlinear uncertainties," Chaos, Solitons and Fractals, vol. 38, no. 1, pp. 160-167, 2008.

[2] C.-C. Shen and S.-M. Zhong, "New delay-dependent robust stability criterion for uncertain neutral systems with time-varying delay and nonlinear uncertainties," Chaos, Solitons and Fractals, vol. 40, no. 5, pp. 2277-2285, 2009.

[3] K.-W. Yu and C.-H. Lien, "Stability criteria for uncertain neutral systems with interval time-varying delays," Chaos, Solitons and Fractals, vol. 38, no. 3, pp. 650-657, 2008. 
[4] O. M. Kwon, J. H. Park, and S. M. Lee, “On delay-dependent robust stability of uncertain neutral systems with interval time-varying delays," Applied Mathematics and Computation, vol. 203, no. 2, pp. 843-853, 2008.

[5] Q.-L. Han and L. Yu, "Robust stability of linear neutral systems with nonlinear parameter perturbations," IEE Proceedings, vol. 151, no. 5, pp. 539-546, 2004.

[6] F. Qiu, B. Cui, and Y. Ji, "Further results on robust stability of neutral system with mixed time-varying delays and nonlinear perturbations," Nonlinear Analysis. Real World Applications, vol. 11, no. 2, pp. 895-906, 2010.

[7] Y.-Y. Cao and J. Lam, "Computation of robust stability bounds for time-delay systems with nonlinear time-varying perturbations," International Journal of Systems Science, vol. 31, no. 3, pp. 359-365, 2000.

[8] J. Cao and J. Wang, "Delay-dependent robust stability of uncertain nonlinear systems with time delay," Applied Mathematics and Computation, vol. 154, no. 1, pp. 289-297, 2004.

[9] Q.-L. Han, "Robust stability for a class of linear systems with time-varying delay and nonlinear perturbations," Computers \& Mathematics with Applications, vol. 47, no. 8-9, pp. 1201-1209, 2004.

[10] X. Jiang and Q.-L. Han, "Delay-dependent robust stability for uncertain linear systems with interval time-varying delay," Automatica, vol. 42, no. 6, pp. 1059-1065, 2006.

[11] O. M. Kwon and J. H. Park, "Exponential stability for time-delay systems with interval time-varying delays and nonlinear perturbations," Journal of Optimization Theory and Applications, vol. 139, no. 2, pp. 277-293, 2008.

[12] O. M. Kwon, J. H. Park, and S. M. Lee, "On robust stability criterion for dynamic systems with timevarying delays and nonlinear perturbations," Applied Mathematics and Computation, vol. 203, no. 2, pp. 937-942, 2008.

[13] S. Lakshmanan, T. Senthilkumar, and P. Balasubramaniam, "Improved results on robust stability of neutral systems with mixed time-varying delays and nonlinear perturbations," Applied Mathematical Modelling, vol. 35, no. 11, pp. 5355-5368, 2011.

[14] J. H. Park, "Novel robust stability criterion for a class of neutral systems with mixed delays and nonlinear perturbations," Applied Mathematics and Computation, vol. 161, no. 2, pp. 413-421, 2005.

[15] V. N. Phat and P. Niamsup, "Stability of linear time-varying delay systems and applications to control problems," Journal of Computational and Applied Mathematics, vol. 194, no. 2, pp. 343-356, 2006.

[16] R. Rakkiyappan, P. Balasubramaniam, and R. Krishnasamy, "Delay dependent stability analysis of neutral systems with mixed time-varying delays and nonlinear perturbations," Journal of Computational and Applied Mathematics, vol. 235, no. 8, pp. 2147-2156, 2011.

[17] K. Gu, V. L. Kharitonov, and J. Chen, Stability of Time-Delay System, Birkhauser, Boston, Mass, USA, 2003.

[18] Z. Zuo and Y. Wang, "New stability criterion for a class of linear systems with time-varying delay and nonlinear perturbations," IEE Proceedings, vol. 153, no. 5, pp. 623-626, 2006.

[19] T.-F. Li and Z.-D. Xu, "Robust stability criteria of neutral systems with time varying delay and nonlinear uncertainties," in Proceedings of the Chinese Control and Decision Conference (CCDC '09), pp. 4056-4060, 2009. 


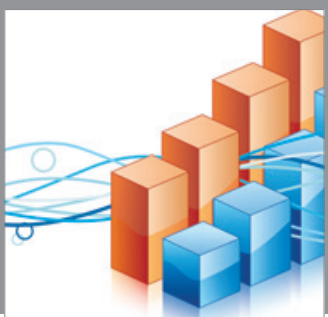

Advances in

Operations Research

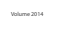

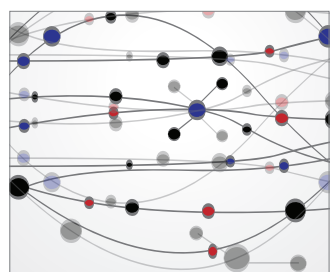

\section{The Scientific} World Journal
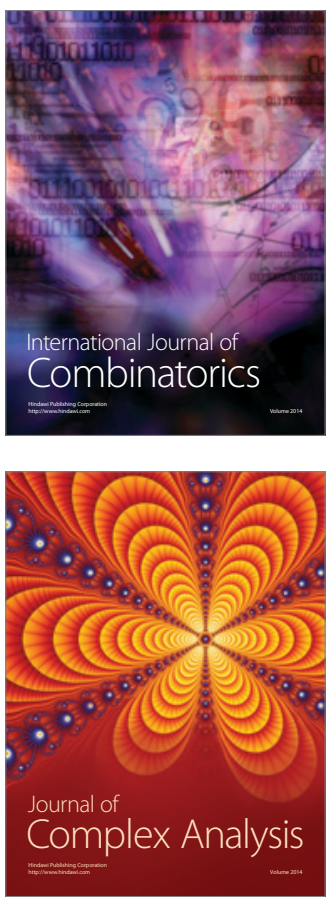

International Journal of

Mathematics and

Mathematical

Sciences
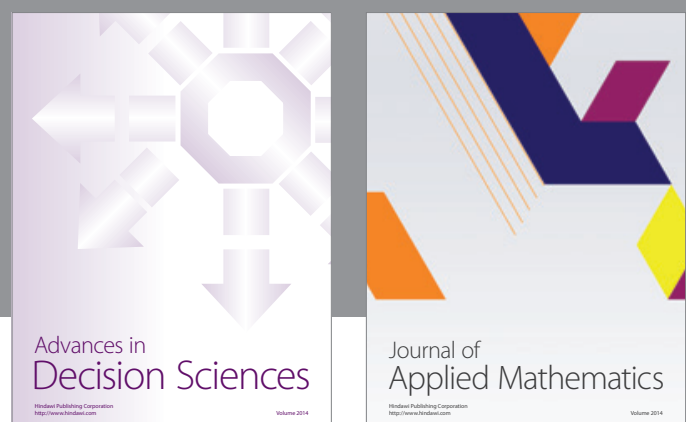

Journal of

Applied Mathematics
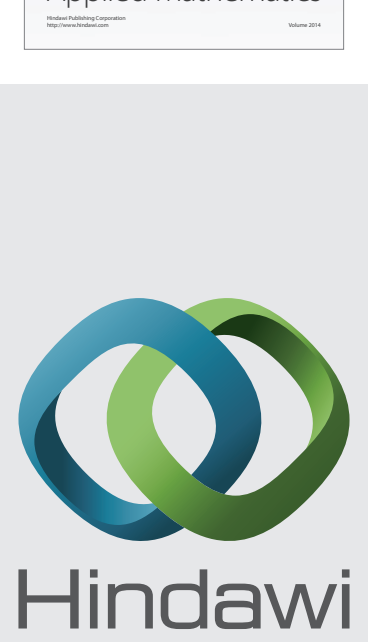

Submit your manuscripts at http://www.hindawi.com
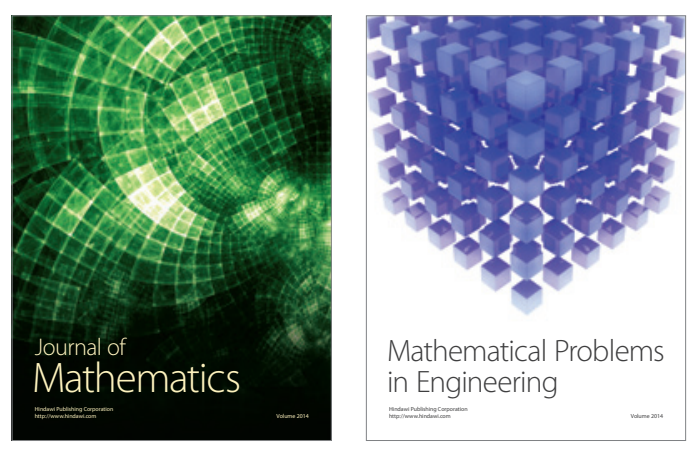

Mathematical Problems in Engineering
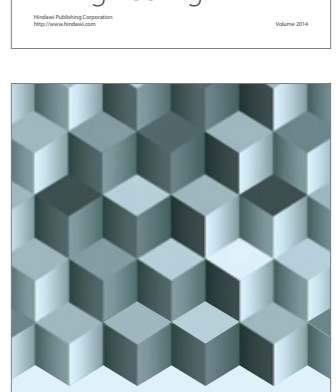

Journal of

Function Spaces
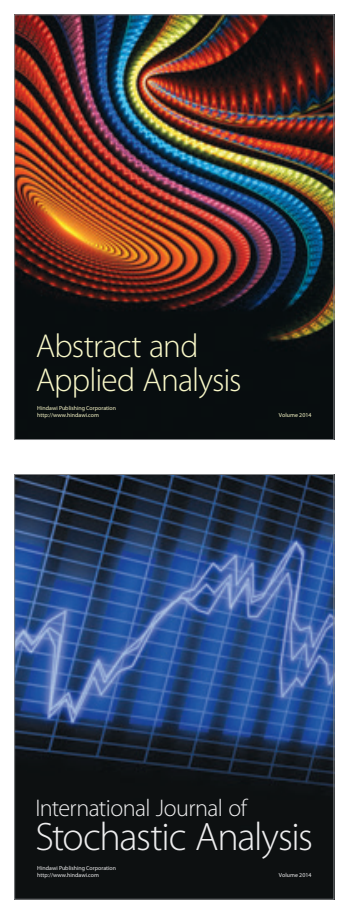

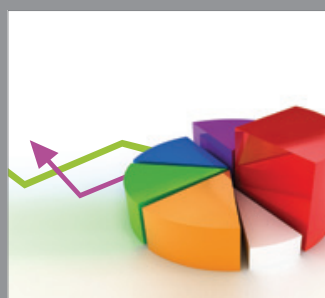

ournal of

Probability and Statistics

Promensencen
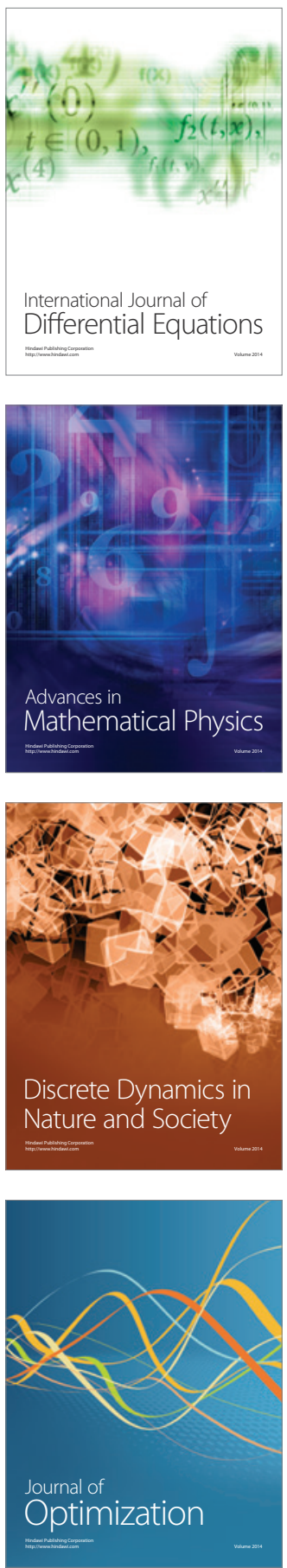